Check for updates

Cite this: Mater. Chem. Front., 2021, 5, 2824

Received 28th November 2020 Accepted 27th January 2021

DOI: 10.1039/d0qm00997k

rsc.li/frontiers-materials

\title{
Covalent functionalization of two-dimensional black phosphorus nanosheets with porphyrins and their photophysical characterization $\dagger$
}

\author{
Shameel Thurakkal and Xiaoyan Zhang (D) *
}

\begin{abstract}
Black phosphorus nanosheets (BPNSs) are a rising star among 2D materials and hold applications in a wide range of research areas. However, the poor stability of BPNSs due to chemical degradation in the presence of air and water limits their practical applications. Chemical functionalization is a promising strategy to improve the stability and impart new properties to BPNSs. Herein, functional porphyrin units are attached onto BPNSs through a direct phosphorus-carbon linkage using diazonium chemistry. The porphyrin functionalized BPNSs are characterized using Raman spectroscopy, X-ray photoelectron spectroscopy (XPS), infrared spectroscopy (IR) and transmission electron microscopy (TEM) analyses. The formation of a $\mathrm{P}-\mathrm{C}$ bond between BPNSs and porphyrin units is confirmed by the appearance of a new peak at $131.16 \mathrm{eV}$ in the high resolution P 2p XPS spectrum. A control experiment under similar conditions with diazonium free porphyrins further supports the covalent attachment by precluding noncovalent interactions between porphyrins and BPNSs in the hybrid. Furthermore, the photophysical properties of the BPNS-TPP hybrid were investigated in detail using steady state and time-resolved spectroscopic techniques. Importantly, the porphyrin functionalized BPNSs exhibit an improved ambient stability compared to pristine BPNSs, confirmed by UV-Vis absorption and XPS measurements. This study proposes a potential useful route to obtain stable functional BPNSs, holding promising applications in optoelectronic devices such as nonlinear optics and solar energy harvesting devices.
\end{abstract}

\section{Introduction}

Over the past few years, as one of the post-graphene era twodimensional (2D) materials, ${ }^{1-7}$ black phosphorus nanosheets (BPNSs) have attracted tremendous attention due to their unique properties such as high charge carrier mobility, thickness dependent tunable direct band gap and strong in-plane anisotropy..$^{8-12} 2 \mathrm{D}$ BPNSs hold potential applications in the fields of photocatalysis, biomedicine, batteries, supercapacitors, field effect transistors (FETs), optoelectronics and sensing devices. ${ }^{13-21}$ However, the poor ambient stability of BPNSs limits their practical application. It was found that BPNSs undergo chemical degradation to phosphorus oxides in the presence of ambient oxygen and/or water due to the high reactivity of the lone pair of electrons of phosphorus atoms. ${ }^{22-25}$ So far, considerable efforts have been expended in improving the stability of BPNSs through $\mathrm{Al}_{2} \mathrm{O}_{3}$ protective layer coating, h-BN

Division of Chemistry and Biochemistry, Department of Chemistry and Chemical Engineering, Chalmers University of Technology, Kemigården 4, SE-412 96 Göteborg, Sweden. E-mail: xiaoyan.zhang@chalmers.se

$\dagger$ Electronic supplementary information (ESI) available. See DOI: 10.1039/ d0qm00997k encapsulation, hybrid $\mathrm{Al}_{2} \mathrm{O}_{3} / \mathrm{BN}$ encapsulation, ionophore coating and chemical functionalization. ${ }^{26-36}$ Among these techniques, chemical modification via covalent or noncovalent strategies opens a broad area of interest not only to passivate but also to explore new chemistry on BPNSs. Covalent functionalization is an effective and promising strategy to passivate BPNSs as this method utilizes the lone pair of electrons of phosphorus atoms for bond formation. Compared with the noncovalent approach, covalent functionalization can provide more stable systems. To this end, different types of reactions such as free radical addition, ${ }^{30,37}$ nitrene addition $^{31}$ and nucleophilic substitution ${ }^{38-40}$ have been developed for covalent functionalization of BPNSs. Using the developed functionalization strategies, integration of novel functional molecules onto BPNSs for improved physicochemical properties is highly desirable. In this context, hybrid materials of BPNSs with multifunctional properties are very few and are not well exploited. ${ }^{41-43}$ Porphyrins are well-known functional dyes with extraordinary physicochemical properties holding versatile applications in photomedicine, and photovoltaic and optoelectronic devices. ${ }^{44-47}$ Porphyrins have been chemically attached onto various nanomaterials including carbon nanotubes, graphene and $\mathrm{MoS}_{2}$ to achieve novel functional hybrid materials. ${ }^{4-52}$ For example, carbon 
nanotube- or graphene-porphyrin hybrid nanomaterials were prepared by covalently attaching porphyrins to carbon nanotubes or graphene oxide via amide bonds. ${ }^{48,50}$ The nanohybrids showed a photoinduced electron and/or energy transfer mechanism and thus, improved optoelectronic performances. In an another report, $\mathrm{MoS}_{2}$ quantum dot (QD)-porphyrin hybrid nanomaterials were achieved by covalently attaching porphyrins to the surface of QDs via a direct C-S linkage. ${ }^{51}$ Graphene-porphyrin hybrid materials have been prepared using the 1,3-dipolar cycloaddition approach on liquid phase exfoliated graphene. ${ }^{52}$ Encouraged by the unique properties of BPNSs and opto-electronically active porphyrins, we designed and synthesized tetraphenyl porphyrin (TPP) functionalized BPNSs (BPNS-TPP) by forming a direct covalent P-C bond using diazonium chemistry. To the best of our knowledge, covalent functionalization of BPNSs with porphyrin molecules and further study of their photophysical properties have not been reported previously.

\section{Experimental}

\section{Materials}

Black phosphorus (BP) was purchased from Smart Elements (purity 99.998\%), and all other chemicals were purchased from Sigma-Aldrich. The bulk BP and solvents were stored in a glovebox. Transfer of solvents and other precursors was carried out in a glovebox, while purification steps after functionalization reactions were performed under a nitrogen atmosphere. The solvents $\mathrm{N}$-methyl-2-pyrrolidone (NMP), acetonitrile and dichloromethane (DCM) are anhydrous and were purchased from Sigma Aldrich. All the solvents were deoxygenated before the experiments unless otherwise mentioned.

\section{Preparation of BPNSs}

Bulk BP (10 mg) was ground and added to anhydrous NMP $(20 \mathrm{~mL})$ in a glovebox. The solution was purged with argon for 15 minutes and then sonicated at $10-15{ }^{\circ} \mathrm{C}$ in a sonication bath for $6 \mathrm{~h}$ under argon protection. After sonication, the mixture was centrifuged at $5000 \mathrm{rpm}$ for 120 minutes and the supernatant was collected to obtain few-layer BPNSs.

\section{Synthesis of TPP- $\mathbf{N}_{2}^{+} \mathrm{BF}_{4}{ }^{-}$}

TPP- $\mathrm{NH}_{2}$ (20 mg, 1 eq.) was added to $\mathrm{HBF}_{4}$ (48 wt\% in $\mathrm{H}_{2} \mathrm{O}$, $0.0132 \mathrm{~mL}, 6.63$ eq.) and acetic acid ( $2 \mathrm{~mL})$ in a three-neck round bottom flask under a nitrogen atmosphere. Isoamyl nitrite ( $0.0133 \mathrm{~mL}, 3.13$ eq.) dissolved in acetic acid $(1 \mathrm{~mL})$ was slowly added into the green solution. After 20 minutes, the reaction mixture was quenched with diethyl ether $(2 \mathrm{~mL})$ and was stored at $-22{ }^{\circ} \mathrm{C}$ for $6 \mathrm{~h}$. The green solid was filtered, washed with an excess amount of diethyl ether and dried in a vacuum (yield: $98 \%$ ).

\section{Synthesis of BPNS-TPP}

BPNSs in NMP $(20 \mathrm{~mL})$ were centrifuged at $12000 \mathrm{rpm}$ for 30 minutes and the residue was re-dispersed in acetonitrile
$(8 \mathrm{~mL})$. The re-dispersed BPNSs were added into a mixture of TPP- $\mathrm{N}_{2}{ }^{+} \mathrm{BF}_{4}{ }^{-}(2 \mathrm{mg}, 0.00275 \mathrm{mmol})$ and tetrabutylammoniumhexafluorophosphate $(1.06 \mathrm{mg}, 0.00275 \mathrm{mmol})$ in acetonitrile $(2 \mathrm{~mL})$ in a round bottom flask protected with argon. The mixture was stirred at room temperature under an argon atmosphere in the dark for $36 \mathrm{~h}$. After that, the solid residue was collected by centrifugation and washed with acetonitrile (3 times) and DCM (5 times) until a clear supernatant was obtained. UV-vis absorption of the clear supernatant was checked, confirming the complete removal of $\mathrm{TPP}-\mathrm{N}_{2}{ }^{+} \mathrm{BF}_{4}{ }^{-}$from the reaction mixture. Finally, the residue was re-dispersed in acetonitrile for further photophysical studies.

\section{Control reaction}

BPNSs in NMP $(20 \mathrm{~mL})$ were centrifuged at $12000 \mathrm{rpm}$ for 30 minutes and the residue was re-dispersed in acetonitrile $(8 \mathrm{~mL})$. The re-dispersed BPNSs were added to a mixture of TPP (2 mg, $0.00324 \mathrm{mmol}$ ) and tetrabutylammoniumhexafluorophosphate $(1.26 \mathrm{mg}, 0.00324 \mathrm{mmol})$ in acetonitrile $(2 \mathrm{~mL})$ in a round bottom flask protected with argon. The mixture was stirred at room temperature under an argon atmosphere in the dark for $36 \mathrm{~h}$. After that, the reaction was stopped, and the mixture was centrifuged at $12000 \mathrm{rpm}$ for 30 minutes. The residue was collected and washed with acetonitrile ( 3 times) and DCM ( 5 times) until a clear supernatant was obtained. Finally, the residue was re-dispersed in acetonitrile for further photophysical studies.

\section{Characterization}

Raman analysis was performed on a WITec alpha300 R confocal Raman microscopy system (excitation at $532 \mathrm{~nm}$ ). X-ray photoelectron spectroscopy (XPS) analysis was carried out on a PHI 5000 VersaProbe III Scanning XPS Microprobe and the spectra were processed using the PHI multiPak software. TEM and HR-TEM were performed on a FEI Tecnai T20 instrument at an acceleration voltage of $200 \mathrm{kV}$. Attenuated total reflectance infrared (ATR-IR) spectra were recorded using a PerkinElmer Frontier Infrared Spectrometer with a GladiATR, diamond crystal design. UV-vis absorption spectra were recorded on a Varian Cary 50 Bio UV-visible spectrophotometer and fluorescence spectra were recorded on a Varian Cary Eclipse fluorescence spectrophotometer. Fluorescence lifetime studies were performed in a time correlated single photon counting (TCSPC) system, EDINBURGH LifeSpec II instrument (excitation wavelength at $405 \mathrm{~nm}) .{ }^{1} \mathrm{H}$ NMR spectra of the molecules were recorded using a $400 \mathrm{MHz}$ Varian NMR spectrometer. Exfoliation of BPNSs was carried out in an Elmasonic $\mathrm{P} 60 \mathrm{H}$ ultrasonic unit at an ultrasonic frequency of $37 \mathrm{kHz}$.

\section{Results and discussion}

The few-layer BPNSs were synthesized by the sonication assisted liquid phase exfoliation method using NMP as the solvent. The diazonium salts of TPP $\left(\mathrm{TPP}-\mathrm{N}_{2}{ }^{+} \mathrm{BF}_{4}{ }^{-}\right)$were synthesized from TPP using a multistep synthetic procedure prior to the functionalization step (Scheme 1a and Schemes S1, 
a)
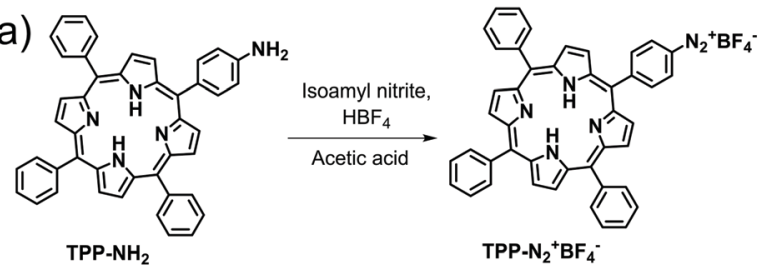

b)
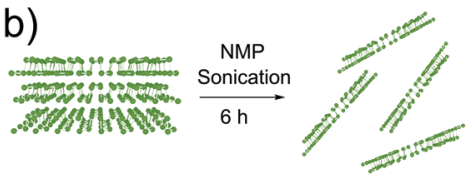

BPNSs

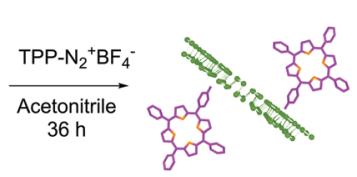

BPNSs-TPP

Scheme 1 (a) Synthesis of TPP $-\mathrm{N}_{2}{ }^{+} \mathrm{BF}_{4}{ }^{-}$. (b) Exfoliation of bulk BP into BPNSs and covalent functionalization of BPNSs using TPP $-\mathrm{N}_{2}{ }^{+} \mathrm{BF}_{4}{ }^{-}$.

$\mathrm{S} 2, \mathrm{ESI} \dagger)$. The porphyrin derivatives were characterized using ${ }^{1} \mathrm{H}$ NMR (Fig. S1 and S2, ESI $\dagger$ ) and the formation of diazonium salt was unambiguously confirmed by the strong vibrational peak at $2273 \mathrm{~cm}^{-1}$ corresponding to the diazo group in the IR spectrum (Fig. S3, ESI $\dagger$ ). Chemical functionalization of BPNSs was performed by reacting the exfoliated BPNSs with TPP$\mathrm{N}_{2}{ }^{+} \mathrm{BF}_{4}{ }^{-}$in acetonitrile (Scheme 1b). After functionalization, the reaction mixture was washed with acetonitrile followed by DCM to remove the physisorbed porphyrins. Before each washing step, the mixture was sonicated for 5 minutes, and the process was repeated to obtain a clear supernatant which did not show any absorption and fluorescence signals from the reactant TPP$\mathrm{N}_{2}{ }^{+} \mathrm{BF}_{4}{ }^{-}$. The exfoliated and functionalized BPNSs were then fully characterized by various spectroscopy and microscopy methods.

Mean Raman spectra were obtained by averaging more than 1000 point Raman spectra, recorded using a $532 \mathrm{~nm}$ excitation laser. As shown in Fig. 1, the BPNSs show three prominent Raman bands related to the out-of-plane phonon mode $\mathrm{A}_{\mathrm{g}}^{1}$ at $358 \mathrm{~cm}^{-1}$, in-plane modes $B_{2 g}$ at $432 \mathrm{~cm}^{-1}$ and $A_{g}^{2}$ at $460 \mathrm{~cm}^{-1}$. After functionalization, the wavenumbers corresponding to $B_{2} g$ and $\mathrm{A}_{\mathrm{g}}^{2}$ bands were found to be redshifted by $3 \mathrm{~cm}^{-1}$ whereas that of the $A_{g}^{1}$ band remains unchanged. The normalized peak intensities (using silicon peak as the reference) of all the three peaks were found to be decreased after functionalization. The decrease of the corresponding Raman intensities of BPNSs is

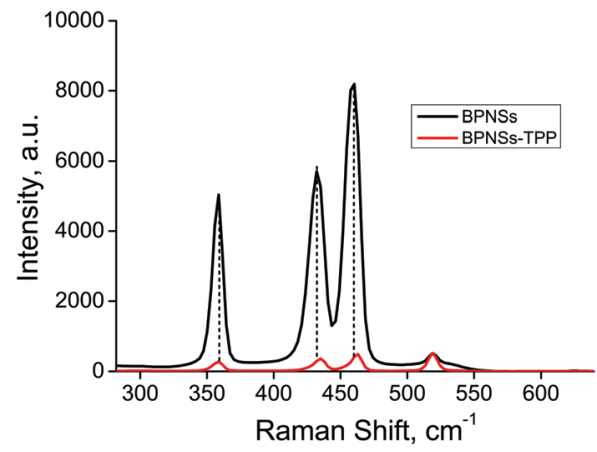

Fig. 1 Mean Raman spectra of BPNSs and BPNSs-TPP measured using a $532 \mathrm{~nm}$ laser excitation.
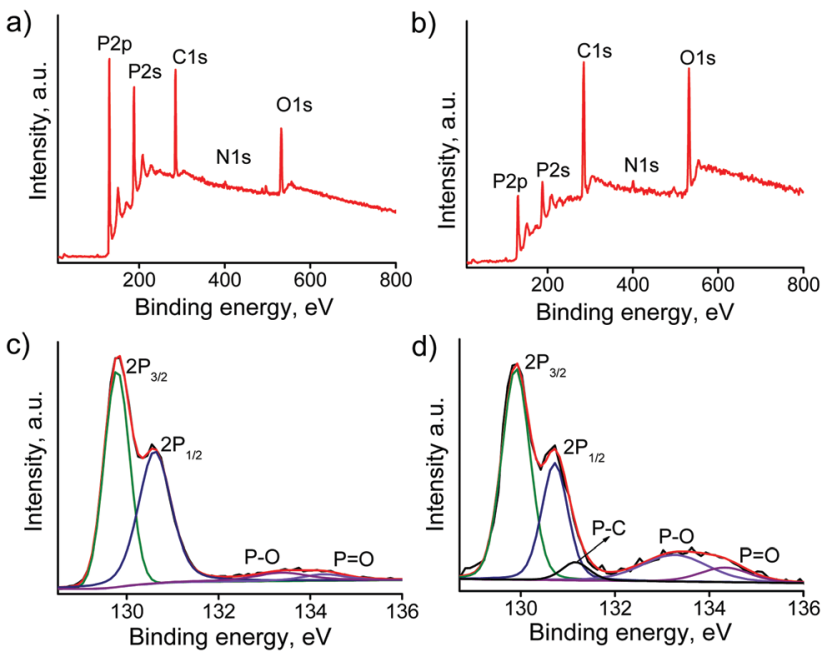

Fig. 2 XPS survey spectra and P 2p HR-XPS spectra of (a and c) BPNSs and ( $b$ and $d$ ) BPNS-TPP, respectively.

attributed to the disruption of intralayer phosphorus bonding after functionalization. ${ }^{30}$ Covalent functionalization of BPNSs was confirmed by high resolution X-ray photoelectron spectroscopy (HR-XPS) in Fig. 2. The P 2p core-level XPS spectrum of BPNSs could be fitted to four peaks at 129.78, 130.61, 133.38 and $134.30 \mathrm{eV}$, assigned to $\mathrm{P} 2 \mathrm{p}_{3 / 2}, \mathrm{P} 2 \mathrm{p}_{1 / 2}, \mathrm{P}-\mathrm{O}$ and $\mathrm{P}=\mathrm{O}$ functionalities. ${ }^{42,53}$ After covalent functionalization of BPNSs with TPP, the P 2p core level XPS spectrum showed five peaks centered at 129.90, 130.72, 131.16, 133.27 and $134.33 \mathrm{eV}$, respectively. The new peak at $131.16 \mathrm{eV}$ in the BPNS-TPP is attributed to the formation of the $\mathrm{P}-\mathrm{C}$ bond between BPNS and porphyrin molecules. The presence of TPP was confirmed by the appearance of two peaks in the $\mathrm{N}$ 1s spectrum (Fig. S5, $\mathrm{ESI} \dagger$ ). One peak is at $399.65 \mathrm{eV}$ and the other one is at $400.25 \mathrm{eV}$, corresponding to $-\mathrm{C}=\mathrm{N}-$ and $-\mathrm{NH}-$ nitrogen, respectively. Based on the fitting of the $\mathrm{P} 2 \mathrm{p}$ spectrum, we can deduce that the degree of functionalization for the BPNS-TPP is $11.6 \mathrm{P}$ atoms each possessing one TPP unit.

Furthermore, we recorded the FTIR spectra of BPNSs and BPNS-TPP using the attenuated total reflectance (ATR) method and compared them with those of TPP (Fig. 3). The BPNS spectra exhibit weak vibrational peaks at 1450 and $1370 \mathrm{~cm}^{-1}$ which can be attributed to the $\mathrm{PO}_{x}$ species formed during the sample preparation. Similar peaks were observed in the BPNSTPP spectra along with clear vibrational signals corresponding to the fingerprint region of porphyrin molecules and a weak absorption at $870 \mathrm{~cm}^{-1}$ due to the $\mathrm{P}-\mathrm{C}$ stretching vibration. ${ }^{42,54}$ The presence of additional peaks in the BPNS-TPP compared to the bare BPNSs and the $\mathrm{P}-\mathrm{C}$ stretching further confirms the successful covalent modification of BPNSs with TPP. The morphology of exfoliated and functionalized BPNSs was studied using transmission electron microscopy (TEM) and high resolution TEM (HR-TEM). The exfoliated BPNSs showed a few-layer sheet like morphology. The BPNS-TPP hybrid also showed a similar structure to the bare BPNSs, suggesting that the morphology of the nanosheets was not affected after 


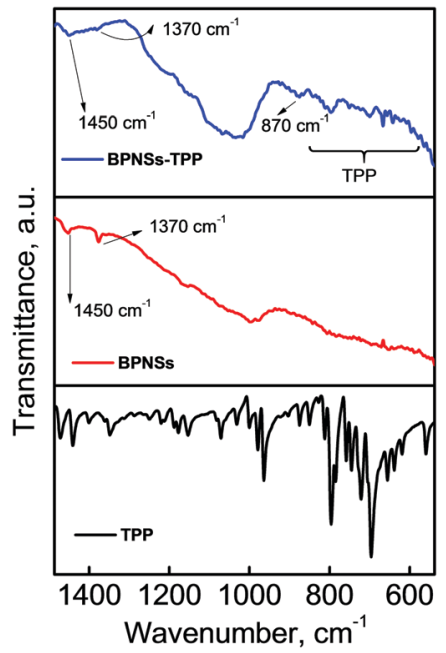

Fig. 3 FTIR spectra of TPP, BPNSs and BPNS-TPP.

covalent functionalization (Fig. 4a, c and Fig. S6a, b and S7a, b, ESI $\dagger$ ). In the HR-TEM images, lattice fringes of the (111) plane with a space distance of about $2.5 \AA$ were observed for both BPNSs and BPNS-TPP indicating that the TPP functionalization does not affect the crystallinity of BPNSs (Fig. 4b, d and Fig. S6c, d and S7c, d, ESI $\dagger$ ).

The UV-vis absorption of BPNSs and BPNS-TPP was measured in acetonitrile and compared with that of TPP (Fig. 5a). TPP exhibits an intense Soret band at $412 \mathrm{~nm}$ and four less intense $\mathrm{Q}$ bands from the 500 to $700 \mathrm{~nm}$ region. Compared with bare BPNSs, the BPNS-TPP shows an absorption peak at $415 \mathrm{~nm}$ corresponding to the Soret band of TPP. The $3 \mathrm{~nm}$ redshift of the absorption peak indicates the electronic interaction of
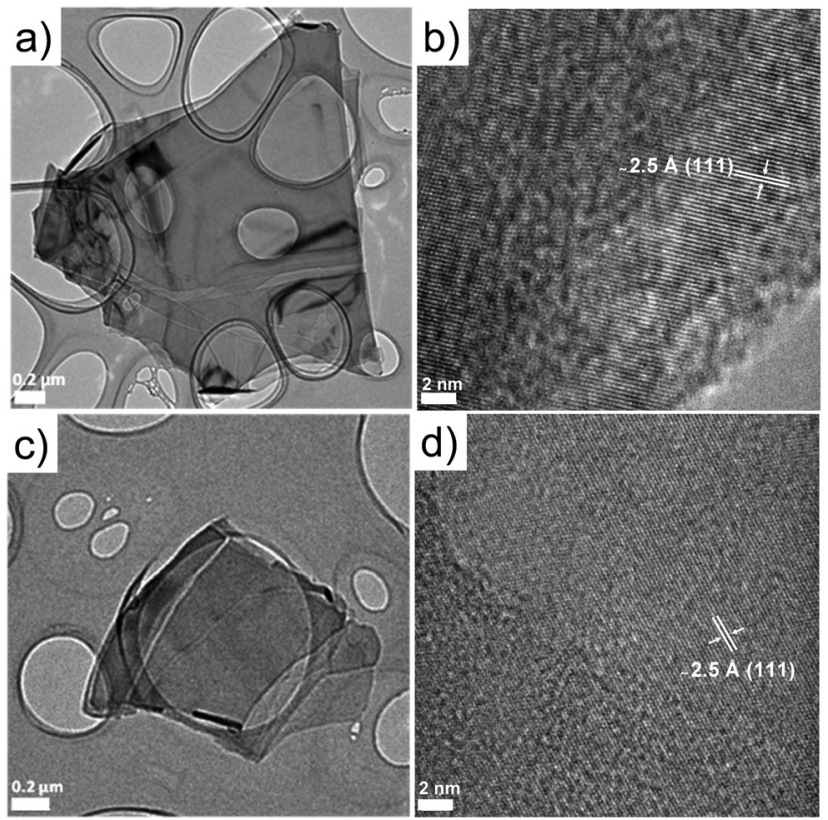

Fig. 4 TEM and HR-TEM images of ( $a$ and $b$ ) exfoliated BPNSs and ( $c$ and d) BPNS-TPP, respectively.
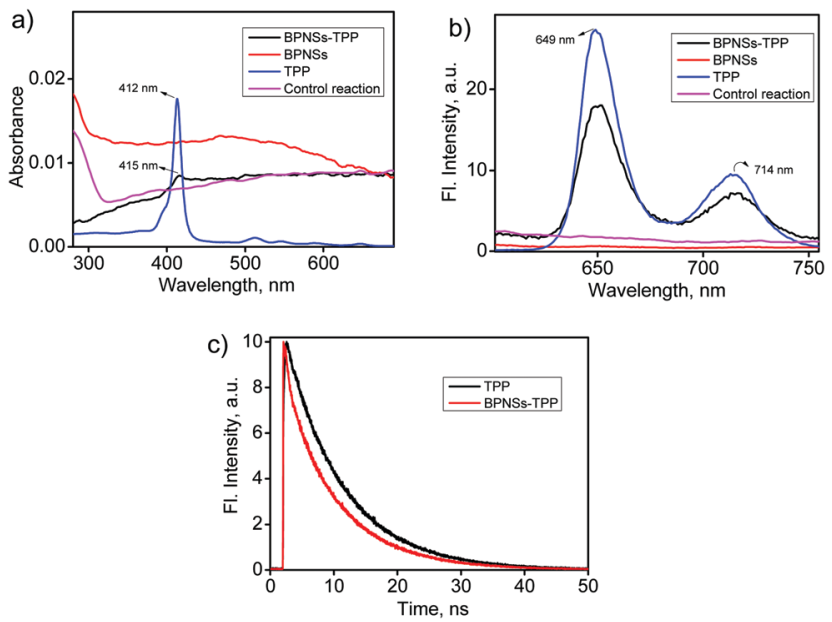

Fig. 5 (a) UV-Vis absorption spectra and (b) fluorescence spectra of BPNSS-TPP, BPNSs, TPP and the control sample in acetonitrile, $\lambda_{\text {exc }}=415 \mathrm{~nm}$. (c) Fluorescence decay profile of TPP and BPNS-TPP in acetonitrile $\left(\lambda_{\text {exc }}=405 \mathrm{~nm}\right)$.

porphyrin molecules with BPNSs. In order to understand the excited state interactions between TPP and BPNSs, the fluorescence spectra of BPNSs, BPNSS-TPP and TPP in acetonitrile were recorded (Fig. 5b). TPP shows two emission peaks at 649 and $714 \mathrm{~nm}$. The fluorescence spectrum of BPNSs-TPP shows emission peaks at 650 and $715 \mathrm{~nm}\left(\lambda_{\mathrm{exc}}=415 \mathrm{~nm}\right)$, corresponding to the fluorescence peaks of TPP. Importantly, we have observed no absorption and fluorescence peaks from TPP$\mathrm{N}_{2}{ }^{+} \mathrm{BF}_{4}^{-}$in the functionalized sample, suggesting the successful grafting of TPP onto BPNSs and the complete removal of unreacted TPP- $\mathrm{N}_{2}{ }^{+} \mathrm{BF}_{4}{ }^{-}$from the solution during the washing process. On the other hand, bare BPNSs show no emission in the region of 600-800 nm upon excitation at $415 \mathrm{~nm}$. Furthermore, the fluorescence intensity of BPNS-TPP was found to be significantly quenched compared with that of TPP upon excitation at $415 \mathrm{~nm}$ (Fig. S9a and b, ESI, $\dagger$ measurements were performed by keeping the absorbance the same at the excitation wavelength). We have also performed a control reaction in which TPP was used instead of TPP- $\mathrm{N}_{2}{ }^{+} \mathrm{BF}_{4}{ }^{-}$while the rest of the reaction conditions was kept the same. The UV-Vis and fluorescence spectra of the resultant control sample were recorded in acetonitrile and peaks corresponding to porphyrins were not observed. The control experiment unequivocally confirms that there are no physisorbed porphyrins on the BPNSs and suggests the covalent linkage of BPNSs and TPP in the BPNS-TPP hybrid.

The fluorescence quantum yield of the BPNS-TPP hybrid was calculated in acetonitrile using TPP as the standard. The fluorescence quantum yield $\left(\Phi_{\mathrm{f}}\right)$ of TPP is $5.9 \%$ while that of the BPNS-TPP hybrid was found to be $3.5 \%{ }^{55}$ The observed lower fluorescence quantum yield of the hybrid compared with the bare TPP can be attributed to the electron/energy transfer between the excited porphyrins and the BPNSs. A similar observation was found in the porphyrin attached graphene and carbon nanotubes where the porphyrin acts as an electron donor and the later acts as an electron acceptor. ${ }^{48,50,56} \mathrm{~A}$ physical mixture of BPNSs with TPP corresponding to the loading amount of TPP in the BPNSs-TPP hybrid showed a 

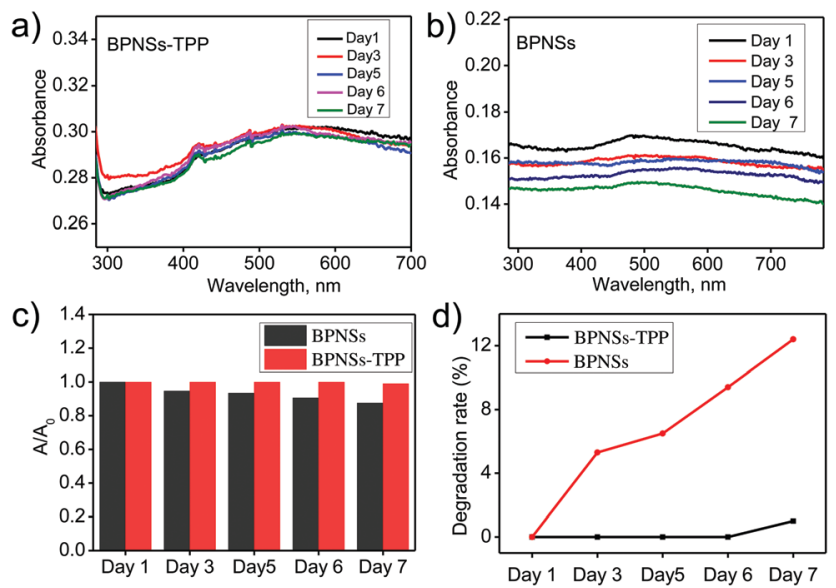

Fig. 6 UV/vis absorption spectra of (a) BPNSs-TPP and (b) pristine BPNSs dispersed in acetonitrile after exposure to air for one week. (c) Variation of the absorption ratios at $480 \mathrm{~nm}\left(A / A_{0}\right)$ and $(d)$ degradation rate $\left(1-A / A_{0}\right)$ of the BPNS-TPP and BPNSs after exposure to ambient conditions for one week.

smaller redshift $(1 \mathrm{~nm})$ in the absorption spectrum and less quenching in the flourescence intensty $\left(\Phi_{\mathrm{f}}=4 \%\right.$ ) (Fig. S9b, ESI $\dagger$ ). Combining the results from the control experiment, the bare BPNSs can have weak noncovalent interactions with TPP which can be distroyed with repeated solvent washing. In order to further support the electronic communication between the TPP units and BPNSs in the BPNS-TPP, fluorescence lifetime measurements were performed by using a time correlated single photon counting (TCSPC) system (Fig. 5c). The fluorescence time profiles showed a biexponential decay kinetics of the photoexcited TPP in the BPNSS-TPP, with a lifetime of $8.49 \mathrm{~ns}(67.6 \%)$ and $0.38 \mathrm{~ns}(32.4 \%)$. On the other hand, a larger lifetime of $8.89 \mathrm{~ns}$ was found for TPP than the hybrid, indicating the electron/energy transfer process in the BPNS-TPP hybrid.

To evaluate the ambient stability of the functionalized BPNSs, we have recorded the changes in the UV-Vis absorption spectra for both BPNSs and BPNS-TPP in non-deoxygenated acetonitrile under ambient conditions (Fig. $6 \mathrm{a}$ and b). The variation of optical absorbance at $480 \mathrm{~nm}$ and degradation rates were calculated and are shown in the Fig. $6 \mathrm{c}$ and d. ${ }^{31}$ BPNSs showed a gradual decrease in the absorbance at $480 \mathrm{~nm}$ because of their instability under ambient conditions and the degradation ratio reaches $12 \%$ after exposure to ambient conditions for one week. Interestingly, the BPNS-TPP showed negligible changes in the absorption spectrum and the degradation ratio decreased to $1 \%$ even after exposure to ambient conditions for one week. This dramatic difference in the optical absorption confirms that the covalent functionalization of BPNSs with porphyrins can largely improve the stability of BPNSs under ambient conditions. Furthermore, HR-XPS was employed to confirm the improved ambient stability of BPNSs after functionalization. The P 2p core-level XPS spectra for both the BPNSs and the BPNS-TPP were recorded after exposure to ambient conditions for one week and the ratio of peak areas under the $\mathrm{P}-\mathrm{Ox}$ region to $\mathrm{P} 2 \mathrm{p}$ region $(\mathrm{P}-\mathrm{Ox} / \mathrm{P} 2 \mathrm{p})$ was compared with that of day 1 spectra (Fig. 7a and b). The bare BPNSs exhibited $\sim 31 \%$ increase in the $\mathrm{P}-\mathrm{Ox} / \mathrm{P} 2 \mathrm{p}$ ratio whereas the
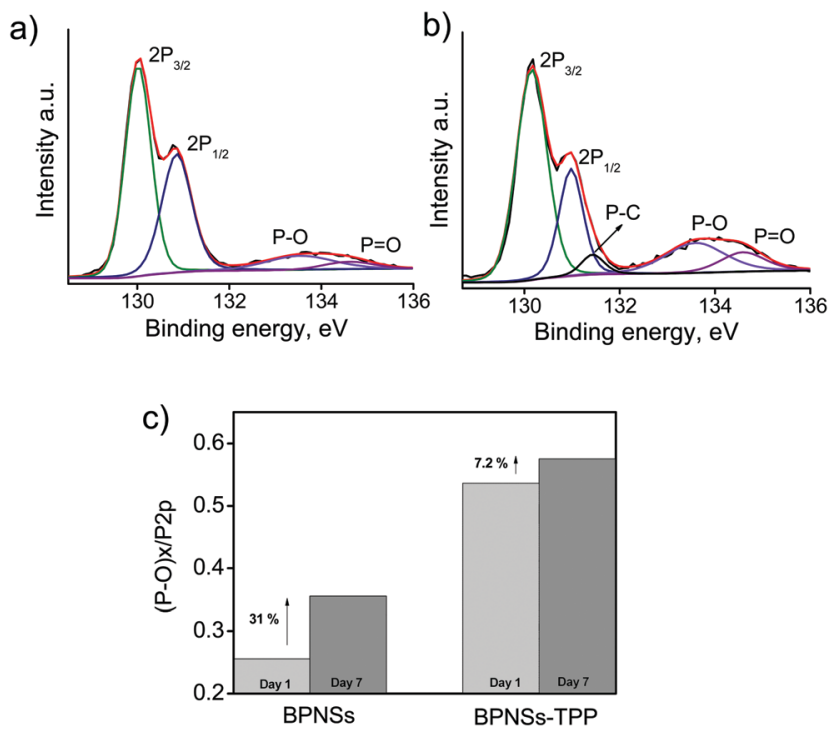

Fig. 7 P 2p HR-XPS spectra of (a) BPNSs and (b) BPNS-TPP after exposure to ambient conditions for one week. (c) Comparison of the ratio of peak areas between the $\mathrm{P}-\mathrm{Ox}$ region and $\mathrm{P} 2 \mathrm{p}$ region $(\mathrm{P}-\mathrm{Ox} / \mathrm{P} 2 \mathrm{p})$ before and after exposure to ambient conditions.

BPNS-TPP showed an increase of only $\sim 7.2 \%$ (Fig. $7 \mathrm{c}$ ). This indicates the effective functionalization and passivation of BPNSs by the TPP molecules.

\section{Conclusions}

In summary, porphyrin covalently modified BPNSs were successfully synthesized by employing diazonium reactions on BPNSs. The covalent nature of the hybrid was confirmed using HR-XPS, Raman, and ATR-IR spectroscopy and further by the control experiment. The effective functionalization was further confirmed by investigating the photophysical properties of the BPNSs and the BPNS-TPP hybrid. The observed fluorescence quenching of the BPNS-TPP hybrid and its fluorescence lifetime profile suggests an electron and/or energy transfer between the porphyrin molecules and the BPNSs. Compared with pristine BPNSs, the BPNS-TPP hybrid showed a dramatic improvement in ambient stability. Our results reveal that the covalent functionalization of porphyrin onto BPNSs is a useful strategy to impart new optoelectronic properties and enhance the ambient stability of BPNSs. It is anticipated that the current findings will fuel the search for multifunctional BPNSs with enhanced ambient stability toward optoelectronic applications such as nonlinear optics and solar energy harvesting devices.

\section{Conflicts of interest}

There are no conflicts to declare.

\section{Acknowledgements}

This work was financially supported by "Stiftelsen Chalmers Tekniska Högskola", "Helge Ax:son Johnsons stiftelse" (F19- 
0050), “Adlerbertska Forskningsstiftelsen” (C 2020-1230), "Göteborg Energi"” (Tänk:Om Stipendiet) and Swedish Research Council Starting Grant (2020-04903).

\section{Notes and references}

1 A. C. Ferrari, F. Bonaccorso, V. Fal'ko, K. S. Novoselov, S. Roche, P. Bøggild, S. Borini, F. H. L. Koppens, V. Palermo, N. Pugno, J. A. Garrido, R. Sordan, A. Bianco, L. Ballerini, M. Prato, E. Lidorikis, J. Kivioja, C. Marinelli, T. Ryhänen, A. Morpurgo, J. N. Coleman, V. Nicolosi, L. Colombo, A. Fert, M. Garcia-Hernandez, A. Bachtold, G. F. Schneider, F. Guinea, C. Dekker, M. Barbone, Z. Sun, C. Galiotis, A. N. Grigorenko, G. Konstantatos, A. Kis, M. Katsnelson, L. Vandersypen, A. Loiseau, V. Morandi, D. Neumaier, E. Treossi, V. Pellegrini, M. Polini, A. Tredicucci, G. M. Williams, B. Hee Hong, J.-H. Ahn, J. Min Kim, H. Zirath, B. J. van Wees, H. van der Zant, L. Occhipinti, A. Di Matteo, I. A. Kinloch, T. Seyller, E. Quesnel, X. Feng, K. Teo, N. Rupesinghe, P. Hakonen, S. R. T. Neil, Q. Tannock, T. Löfwander and J. Kinaret, Science and technology roadmap for graphene, related twodimensional crystals, and hybrid systems, Nanoscale, 2015, 7, 4598-4810.

2 J. Zhang, G. Chen, K. Müllen and X. Feng, Carbon-Rich Nanomaterials: Fascinating Hydrogen and Oxygen Electrocatalysts, Adv. Mater., 2018, 30, 1800528.

3 Y. Wang, Y. Li and Z. Chen, Planar Hypercoordinate Motifs in Two-Dimensional Materials, Acc. Chem. Res., 2020, 53, 887-895.

4 C. Tan, X. Cao, X.-J. Wu, Q. He, J. Yang, X. Zhang, J. Chen, W. Zhao, S. Han, G.-H. Nam, M. Sindoro and H. Zhang, Recent Advances in Ultrathin Two-Dimensional Nanomaterials, Chem. Rev., 2017, 117, 6225-6331.

5 J. Zhu, C. Yang, C. Lu, F. Zhang, Z. Yuan and X. Zhuang, Two-Dimensional Porous Polymers: From Sandwich-like Structure to Layered Skeleton, Acc. Chem. Res., 2018, 51, 3191-3202.

6 Y. Liu, X. Duan, Y. Huang and X. Duan, Two-dimensional transistors beyond graphene and TMDCs, Chem. Soc. Rev., 2018, 47, 6388-6409.

7 S. Z. Butler, S. M. Hollen, L. Cao, Y. Cui, J. A. Gupta, H. R. Gutiérrez, T. F. Heinz, S. S. Hong, J. Huang, A. F. Ismach, E. Johnston-Halperin, M. Kuno, V. V. Plashnitsa, R. D. Robinson, R. S. Ruoff, S. Salahuddin, J. Shan, L. Shi, M. G. Spencer, M. Terrones, W. Windl and J. E. Goldberger, Progress, Challenges, and Opportunities in Two-Dimensional Materials Beyond Graphene, ACS Nano, 2013, 7, 2898-2926.

8 S. Thurakkal and X. Y. Zhang, Recent Advances in Chemical Functionalization of 2D Black Phosphorous Nanosheets, Adv. Sci., 2020, 7, 1902359.

9 V. Eswaraiah, Q. Zeng, Y. Long and Z. Liu, Black Phosphorus Nanosheets: Synthesis, Characterization and Applications, Small, 2016, 12, 3480-3502.
10 S. Thurakkal, D. Feldstein, R. Perea-Causín, E. Malic and X. Y. Zhang, The Art of Constructing Black Phosphorus Nanosheet Based Heterostructures: From 2D to 3D, Adv. Mater., 2021, 33, 2005254.

11 N. Mao, J. Tang, L. Xie, J. Wu, B. Han, J. Lin, S. Deng, W. Ji, H. Xu, K. Liu, L. Tong and J. Zhang, Optical Anisotropy of Black Phosphorus in the Visible Regime, J. Am. Chem. Soc., 2016, 138, 300-305.

12 P. Allongue, M. Delamar, B. Desbat, O. Fagebaume, R. Hitmi, J. Pinson and J.-M. Savéant, Covalent Modification of Carbon Surfaces by Aryl Radicals Generated from the Electrochemical Reduction of Diazonium Salts, J. Am. Chem. Soc., 1997, 119, 201-207.

13 B. Li, C. Lai, G. Zeng, D. Huang, L. Qin, M. Zhang, M. Cheng, X. Liu, H. Yi, C. Zhou, F. Huang, S. Liu and Y. Fu, Black Phosphorus, a Rising Star 2D Nanomaterial in the Post-Graphene Era: Synthesis, Properties, Modifications, and Photocatalysis Applications, Small, 2019, 15, 1804565.

14 X. X. Ge, Z. H. Xia and S. J. Guo, Recent Advances on Black Phosphorus for Biomedicine and Biosensing, Adv. Funct. Mater., 2019, 29, 1900318.

15 S. Wu, K. S. Hui and K. N. Hui, 2D Black Phosphorus: from Preparation to Applications for Electrochemical Energy Storage, Adv. Sci., 2018, 5, 1700491.

16 H. Liu, K. Hu, D. Yan, R. Chen, Y. Zou, H. Liu and S. Wang, Recent Advances on Black Phosphorus for Energy Storage, Catalysis, and Sensor Applications, Adv. Mater., 2018, 30, 1800295.

17 S.-Y. Cho, H.-J. Koh, H.-W. Yoo and H.-T. Jung, Tunable Chemical Sensing Performance of Black Phosphorus by Controlled Functionalization with Noble Metals, Chem. Mater., 2017, 29, 7197-7205.

18 C. Han, Z. Hu, L. C. Gomes, Y. Bao, A. Carvalho, S. J. R. Tan, B. Lei, D. Xiang, J. Wu, D. Qi, L. Wang, F. Huo, W. Huang, K. P. Loh and W. Chen, Surface Functionalization of Black Phosphorus via Potassium toward High-Performance Complementary Devices, Nano Lett., 2017, 17, 4122-4129.

19 F. Liu, C. Huang, C.-X. Liu, R. Shi and Y. Chen, Black Phosphorus-Based Semiconductor Heterojunctions for Photocatalytic Water Splitting, Chem. - Eur. J., 2020, 26, 4449-4460.

20 Y. Liu, J. N. B. Rodrigues, Y. Z. Luo, L. Li, A. Carvalho, M. Yang, E. Laksono, J. Lu, Y. Bao, H. Xu, S. J. R. Tan, Z. Qiu, C. H. Sow, Y. P. Feng, A. H. C. Neto, S. Adam, J. Lu and K. P. Loh, Tailoring sample-wide pseudo-magnetic fields on a graphene-black phosphorus heterostructure, Nat. Nanotechnol., 2018, 13, 828-834.

21 J. Shao, C. Ruan, H. Xie, Z. Li, H. Wang, P. K. Chu and X.-F. Yu, Black-Phosphorus-Incorporated Hydrogel as a Sprayable and Biodegradable Photothermal Platform for Postsurgical Treatment of Cancer, Adv. Sci., 2018, 5, 1700848.

22 T. Zhang, Y. Wan, H. Xie, Y. Mu, P. Du, D. Wang, X. Wu, H. Ji and L. Wan, Degradation Chemistry and Stabilization of Exfoliated Few-Layer Black Phosphorus in Water, J. Am. Chem. Soc., 2018, 140, 7561-7567. 
23 G. X. Wang, W. J. Slough, R. Pandey and S. P. Karna, Degradation of phosphorene in air: understanding at atomic level, 2D Mater., 2016, 3, 025011.

24 Q. Zhou, Q. Chen, Y. Tong and J. Wang, Light-Induced Ambient Degradation of Few-Layer Black Phosphorus: Mechanism and Protection, Angew. Chem., Int. Ed., 2016, 55, 11437-11441.

25 Y. Huang, J. Qiao, K. He, S. Bliznakov, E. Sutter, X. Chen, D. Luo, F. Meng, D. Su, J. Decker, W. Ji, R. S. Ruoff and P. Sutter, Interaction of Black Phosphorus with Oxygen and Water, Chem. Mater., 2016, 28, 8330-8339.

26 J. J. Pei, X. Gai, J. Yang, X. B. Wang, Z. F. Yu, D. Y. Choi, B. Luther-Davies and Y. R. Lu, Producing air-stable monolayers of phosphorene and their defect engineering, Nat. Commun., 2016, 7, 10450.

27 J. D. Wood, S. A. Wells, D. Jariwala, K. S. Chen, E. Cho, V. K. Sangwan, X. Liu, L. J. Lauhon, T. J. Marks and M. C. Hersam, Effective passivation of exfoliated black phosphorus transistors against ambient degradation, Nano Lett., 2014, 14, 6964-6970.

28 X. Chen, Y. Wu, Z. Wu, Y. Han, S. Xu, L. Wang, W. Ye, T. Han, Y. He, Y. Cai and N. Wang, High-quality sandwiched black phosphorus heterostructure and its quantum oscillations, Nat. Commun., 2015, 6, 7315.

29 N. Clark, L. Nguyen, M. J. Hamer, F. Schedin, E. A. Lewis, E. Prestat, A. Garner, Y. Cao, M. Zhu, R. Kashtiban, J. Sloan, D. Kepaptsoglou, R. V. Gorbachev and S. J. Haigh, Scalable Patterning of Encapsulated Black Phosphorus, Nano Lett., 2018, 18, 5373-5381.

30 C. R. Ryder, J. D. Wood, S. A. Wells, Y. Yang, D. Jariwala, T. J. Marks, G. C. Schatz and M. C. Hersam, Covalent functionalization and passivation of exfoliated black phosphorus via aryl diazonium chemistry, Nat. Chem., 2016, 8, 597-602.

31 Y. Liu, P. Gao, T. Zhang, X. Zhu, M. Zhang, M. Chen, P. Du, G. W. Wang, H. Ji, J. Yang and S. Yang, Azide Passivation of Black Phosphorus Nanosheets: Covalent Functionalization Affords Ambient Stability Enhancement, Angew. Chem., Int. Ed., 2019, 58, 1479-1483.

32 X. Zhu, T. Zhang, D. Jiang, H. Duan, Z. Sun, M. Zhang, H. Jin, R. Guan, Y. Liu, M. Chen, H. Ji, P. Du, W. Yan, S. Wei, Y. Lu and S. Yang, Stabilizing black phosphorus nanosheets via edge-selective bonding of sacrificial C60 molecules, Nat. Commun., 2018, 9, 4177.

33 Y. Zhao, H. Wang, H. Huang, Q. Xiao, Y. Xu, Z. Guo, H. Xie, J. Shao, Z. Sun, W. Han, X. F. Yu, P. Li and P. K. Chu, Surface Coordination of Black Phosphorus for Robust Air and Water Stability, Angew. Chem., Int. Ed., 2016, 55, 5003-5007.

34 Z. Guo, S. Chen, Z. Wang, Z. Yang, F. Liu, Y. Xu, J. Wang, Y. Yi, H. Zhang, L. Liao, P. K. Chu and X. F. Yu, Metal-IonModified Black Phosphorus with Enhanced Stability and Transistor Performance, Adv. Mater., 2017, 29, 1703811.

35 H. Wang, K. Hu, Z. Li, C. Wang, M. Yu, Z. Li and Z. Li, Black Phosphorus Nanosheets Passivation Using a Tripeptide, Small, 2018, 14, 1801701.

36 E. Passaglia, F. Cicogna, F. Costantino, S. Coiai, S. Legnaioli, G. Lorenzetti, S. Borsacchi, M. Geppi, F. Telesio, S. Heun,
A. Ienco, M. Serrano-Ruiz and M. Peruzzini, Polymer-Based Black Phosphorus (bP) Hybrid Materials by in Situ Radical Polymerization: An Effective Tool To Exfoliate bP and Stabilize bP Nanoflakes, Chem. Mater., 2018, 30, 2036-2048.

37 M. van Druenen, F. Davitt, T. Collins, C. Glynn, C. O’Dwyer, J. D. Holmes and G. Collins, Covalent Functionalization of Few-Layer Black Phosphorus Using Iodonium Salts and Comparison to Diazonium Modified Black Phosphorus, Chem. Mater., 2018, 30, 4667-4674.

38 Z. Sofer, J. Luxa, D. Bouša, D. Sedmidubský, P. Lazar, T. Hartman, H. Hardtdegen and M. Pumera, The Covalent Functionalization of Layered Black Phosphorus by Nucleophilic Reagents, Angew. Chem., Int. Ed., 2017, 56, 9891-9896.

39 S. Wild, M. Fickert, A. Mitrovic, V. Lloret, C. Neiss, J. A. Vidal-Moya, M. Á. Rivero-Crespo, A. Leyva-Pérez, K. Werbach, H. Peterlik, M. Grabau, H. Wittkämper, C. Papp, H.-P. Steinrück, T. Pichler, A. Görling, F. Hauke, G. Abellán and A. Hirsch, Lattice Opening upon Bulk Reductive Covalent Functionalization of Black Phosphorus, Angew. Chem., Int. Ed., 2019, 58, 5763-5768.

40 L. Zhang, L.-F. Gao, L. Li, C.-X. Hu, Q.-Q. Yang, Z.-Y. Zhu, R. Peng, Q. Wang, Y. Peng, J. Jin and H.-L. Zhang, Negatively charged 2D black phosphorus for highly efficient covalent functionalization, Mater. Chem. Front., 2018, 2, 1700-1706.

41 X. Zeng, M. Luo, G. Liu, X. Wang, W. Tao, Y. Lin, X. Ji, L. Nie and L. Mei, Polydopamine-Modified Black Phosphorous Nanocapsule with Enhanced Stability and Photothermal Performance for Tumor Multimodal Treatments, Adv. Sci., 2018, 5, 1800510.

42 Y. Cao, X. Tian, J. Gu, B. Liu, B. Zhang, S. Song, F. Fan and Y. Chen, Covalent Functionalization of Black Phosphorus with Conjugated Polymer for Information Storage, Angew. Chem., Int. Ed., 2018, 57, 4543-4548.

43 Y. T. Zhao, L. P. Tong, Z. B. Li, N. Yang, H. D. Fu, L. Wu, H. D. Cui, W. H. Zhou, J. H. Wang, H. Y. Wang, P. K. Chu and X. F. Yu, Stable and Multifunctional Dye-Modified Black Phosphorus Nanosheets for Near-Infrared Imaging-Guided Photothermal Therapy, Chem. Mater., 2017, 29, 7131-7139.

44 H. Huang, W. Song, J. Rieffel and J. F. Lovell, Emerging applications of porphyrins in photomedicine, Front. Phys., 2015, 3, 1-15.

45 A. Ambroise, R. W. Wagner, P. D. Rao, J. A. Riggs, P. Hascoat, J. R. Diers, J. Seth, R. K. Lammi, D. F. Bocian, D. Holten and J. S. Lindsey, Design and synthesis of porphyrin-based optoelectronic gates, Chem. Mater., 2001, 13, 1023-1034.

46 L. L. Li and E. W. Diau, Porphyrin-sensitized solar cells, Chem. Soc. Rev., 2013, 42, 291-304.

47 S. C. Karunakaran, P. S. Babu, B. Madhuri, B. Marydasan, A. K. Paul, A. S. Nair, K. S. Rao, A. Srinivasan, T. K. Chandrashekar, M. Rao, Ch, R. Pillai and D. Ramaiah, In vitro demonstration of apoptosis mediated photodynamic activity and NIR nucleus imaging through a novel porphyrin, ACS Chem. Biol., 2013, 8, 127-132.

48 D. Baskaran, J. W. Mays, X. P. Zhang and M. S. Bratcher, Carbon nanotubes with covalently linked porphyrin 
antennae: photoinduced electron transfer, J. Am. Chem. Soc., 2005, 127, 6916-6917.

49 D. Dasler, R. A. Schafer, M. B. Minameyer, J. F. Hitzenberger, F. Hauke, T. Drewello and A. Hirsch, Direct Covalent Coupling of Porphyrins to Graphene, J. Am. Chem. Soc., 2017, 139, 11760-11765.

50 Y. F. Xu, Z. B. Liu, X. L. Zhang, Y. Wang, J. G. Tian, Y. Huang, Y. F. Ma, X. Y. Zhang and Y. S. Chen, A Graphene Hybrid Material Covalently Functionalized with Porphyrin: Synthesis and Optical Limiting Property, Adv. Mater., 2009, 21, 1275-1279.

51 P. Jiang, B. Zhang, Z. Liu and Y. Chen, MoS2 quantum dots chemically modified with porphyrin for solid-state broadband optical limiters, Nanoscale, 2019, 11, 20449-20455.

52 X. Y. Zhang, L. Hou, A. Cnossen, A. C. Coleman, O. Ivashenko, P. Rudolf, B. J. van Wees, W. R. Browne and B. L. Feringa, OnePot Functionalization of Graphene with Porphyrin through Cycloaddition Reactions, Chem. - Eur. J., 2011, 17, 8957-8964.
53 X. Zhu, T. Zhang, Z. Sun, H. Chen, J. Guan, X. Chen, H. Ji, P. Du and S. Yang, Black Phosphorus Revisited: A Missing Metal-Free Elemental Photocatalyst for Visible Light Hydrogen Evolution, Adv. Mater., 2017, 29, 1605776.

54 Y. Cao, B. Zhang, X. Tian, M. Gu and Y. Chen, Direct covalent modification of black phosphorus quantum dots with conjugated polymers for information storage, Nanoscale, 2019, 11, 3527-3533.

55 J. R. Darwent, P. Douglas, A. Harriman, G. Porter and M. C. Richoux, Metal Phthalocyanines and Porphyrins as Photosensitizers for Reduction of Water to Hydrogen, Coord. Chem. Rev., 1982, 44, 83-126.

56 N. Karousis, A. S. D. Sandanayaka, T. Hasobe, S. P. Economopoulos, E. Sarantopoulou and N. Tagmatarchis, Graphene oxide with covalently linked porphyrin antennae: Synthesis, characterization and photophysical properties, J. Mater. Chem., 2011, 21, 109-117. 